
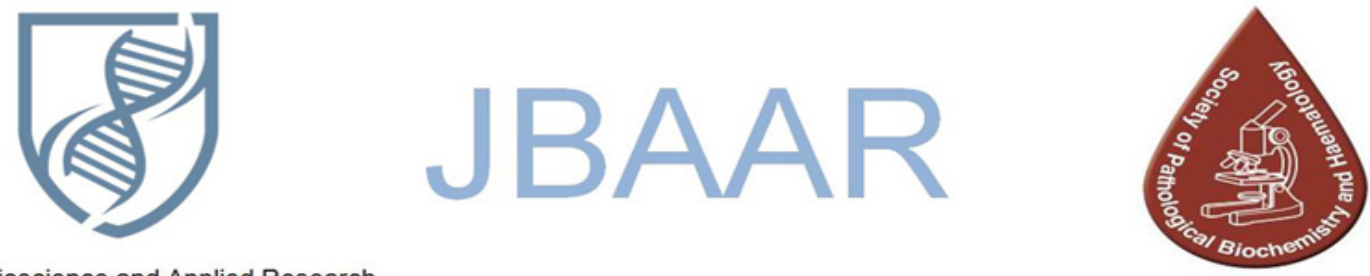

\title{
Immunological study for Helicobacter pylori bacteria in Egyptian patients
}

\author{
Mohamed H. Yassin ${ }^{1}$, Samuel T. MeleK, ${ }^{2}$ Mohamed E. Rashed, ${ }^{3}$ Taghreed A. Zidan ${ }^{1}$ \\ 1.Botany Department, Faculty of Science Benha University, Benha, Egypt. \\ 2.Parasitology and Blood Research Department, National Organization for Drug Control and Research (NODCAR), \\ Cairo, Egypt. \\ 3.Microbiology Department, National organization for Research and Control of Biologicals (NORCB),Cairo, Egypt. \\ Botany Department, Faculty of Science Benha University, Benha, Egypt.
}

(Corresponding author e,mail. totyzedan@gmail.com)

\begin{abstract}
Helicobacter pylori (H. Pylori) are a helix shaped, microaerophilic, Gram-negative, flagellated bacteria. H. Pylori and mankind have an ancient relationship for at least 50,000 years. Commonly the first noninvasively testes used for $\mathrm{H}$. pylori infection's detection were immunological tests like blood antibody test and stool antigen test. We investigated the more efficient susceptibility immunological test for detection of H.pylori infection in adult Egyptian patients by comparing the results of H. Pylori IgA (HpIgA), IgG in serum blood (HpIgG) and H.pylori $\mathrm{Ag}$ in stool (HpSAg) for 30 adult patient against control group using ELISA technique. The results showed that each test could be used successfully for diagnosis of $\mathrm{H}$. pylori. Finally $\mathrm{HpSAg}$ showed reliable results with high sensitivity (> 95\%) followed by HpIgG (87.5\%), while HpIgA showed the lowest sensitivity (37.5\%). Our findings confirms the use of the mentioned immunological tests for detecting them $\mathrm{H}$. Pylori infection and suggest the use $\mathrm{H}$. Pylori $\mathrm{Ag}$ in stool as the most economic, sensitive and reliable method alone or followed by IgG antibody test as confirmatory test to be the first choices for early diagnosis of $\mathrm{H}$. Pylori especially in developing countries.
\end{abstract}

Keywords: Helicobacter pylori, immunological test, IgA,IgG, patients

\section{Introduction}

In 1983 Helicobacter pylori was first isolated from the human stomach by Warren and Marshall (Atherton and
Blaser, 2009). Since then this bacterium became one of the most important human pathogens, infecting more than $50 \%$ of the human population with high prevalence in developing countries(Marshall and Warren, 1984), Helicobacter pylori normally infect stomach, typically during childhood and persists for life (Kusters et al, 2006). However, over $80 \%$ of individuals infected with the bacterium are asymptomatic (Blaser 2006). The infection can lead to peptic ulcer, gastritis, and gastric cancer.Thus, being recognized as the principal agent leading to gastric cancer. World Health Organization (WHO) has classified H. Pylori as a class I carcinogen.

Attributed to the poor socioeconomic status and overcrowded conditions, the lifetime risk of infection is $90 \%$ in third world countries and much less in the developed world. Infection in developed countries is less common in young children and reaches up to $60 \%$ in older ages (Bardhan and Vielt, 1997, Frenck and Clemens, 2003). A study in Egypt revealed that about $91.7 \%$ has been found to be infected in this Egyptian population. The rate of infection was different in different age groups with an increasing trend in older ages and suggested that the rate was increasing in rural areas of this country which make it a public-health issue (El Dine , 2008). Testing for $\mathrm{H}$. pylori is recommended if there is peptic ulcer disease, low grade gastric MALT lymphoma, after endoscopic resection of early gastric cancer, if there are first degree relatives with gastric cancer, and in certain cases of dyspepsia (Marshall et al., 2008). Many diagnostic tests could be used to detect $\mathrm{H}$. Pylori include endoscopic and non- endoscopic methods. The techniques used may be direct (culture, microscopic demonstration of the organism) or indirect (using 
urease, stool antigen, or an antibody response as a marker of disease). Commonly the first noninvasively testes used for $\mathrm{H}$. pylori infection's detection were immunological tests as they are commercially available, easy to perform and inexpensive. Studies showed that infection was associated with a specific gastric immunoglobulin G (IgG) and immunoglobulin A (IgA) response to the bacterium (Rathbone et al, 1985).We investigated the more efficient dependable immunological test for detection of H.pylori infection by comparing the results of H. Pylori IgA (HpIgA), IgG (HpIgG) in serum blood and H.pylori Ag in stool (HpSAg) in adult Egyptian patients

\section{Materials and Methods}

This study was conducted including 50 individuals, A group of 30 patients diagnosed by H.pylori infection, The patients were 35 to 55 years old with a median age of 39 years old ( 39.5 males and 38.5 females). The control group consisted of 20 apparently healthy volunteers from 30-50 years old with median age of 42 years old (44 males and 37 female) with no history of previously been treated of gastric or duodenal ulcer.

Blood drawn for serological testing was performed where $5 \mathrm{ml}$ venous blood was taken from both groups collected in dry tube, after clotting, the sera were separated by centrifugation for (10 minutes at $3000 \mathrm{rpm}$ ) divided into aliquots and stored at $\left(-20^{\circ} \mathrm{C}\right)$ until used. Samples of stools from all individuals in each group were collected in dry clean tubes and stored at $\left(-20^{\circ} \mathrm{C}\right)$ until used. ELISA testing was performed using H.Pylori Antigen E32-320, H. pylori IgG E30-145 and H. pylori IgA E30-274 manufacturer's instructions (Immunospec, USA). Assay values thus calculated for each kit were interpreted as positive, negative according to the manufacturer's instruction as shown in table 1.

\section{Statistical analysis}

The efficacy of the tests was determined by calculating the sensitivity, specificity, Positive predictive value (PPV) and Negative predictive value (NPV) of each test. Statistical analyses were performed using SPSS software (version 16). Chi-square test and independent t-test for significance used to compare proportions and mean between qualitative parameters. The result considered significant if the probability (P-value) was $<0.05$.

Table 1: The parameters used for interpretation of the results according to the ELISA manufacturer's instructions

\begin{tabular}{|c|c|c|c|}
\hline & $\begin{array}{c}\text { H.Pylori Antigen } \\
\text { (HpSAg) }\end{array}$ & $\begin{array}{l}\text { H. pylori IgG } \\
\text { (HpIgG) }\end{array}$ & $\begin{array}{l}\text { H. pylori IgA } \\
\text { (HpIgA) }\end{array}$ \\
\hline Negative & $<15 \mathrm{ng} / \mathrm{ml}$ & \multicolumn{2}{|c|}{$<0.9 *$} \\
\hline Positive & $>20 \mathrm{ng} / \mathrm{ml}$ & \multicolumn{2}{|c|}{$>1.1^{*}$} \\
\hline Indeterminate & $15-20 \mathrm{ng} / \mathrm{ml}$ & \multicolumn{2}{|c|}{$0.9-1.1^{*}$} \\
\hline
\end{tabular}

*ratio between the average absorbance value of sample and that of the cut- off.

\section{Results}

Of 50 patients, only 36 serums' specimens were tested for (HpIgG) and (HpIgA) while all 50 stools' specimens were tested for (HpSAg) as shown in the table 2. The rest of patient refused to undergo the serological tests.

Table 2: Diagnosis test for Helicobacter pylori in control and patient groups Patient group

\begin{tabular}{|c|c|c|c|c|}
\hline \multirow{2}{*}{\begin{tabular}{c} 
Measurement \\
\cline { 2 - 4 }
\end{tabular}} & \multicolumn{2}{|c|}{$\begin{array}{c}\text { Control group } \\
\text { 20) Cases ( }\end{array}$} & \multicolumn{2}{c|}{$\begin{array}{c}\text { Patient group } \\
\text { Cases ( 30) }\end{array}$} \\
\cline { 2 - 5 } & Female (10) & Male (10) & Female (14) & Male (16) \\
\hline $\begin{array}{c}\text { H. Pylori IgA antibody in serum } \\
\text { (HpIgA) }\end{array}$ & 10 & 10 & 6 & 10 \\
\hline $\begin{array}{c}\text { H.pylori IgG antibody in serum } \\
\text { (HpIgG) }\end{array}$ & 10 & 10 & 6 & 16 \\
\hline $\begin{array}{c}\text { H.pylori Antigen in stool } \\
\text { (HpSAg) }\end{array}$ & 10 & 10 & 14 & \\
\hline
\end{tabular}


For control group all tests performed showed negative results expressed by mean (minimum, maximum): 0.346 (0.11, 0.82) for HpIgA, 0.492 (0.142, 0.89) for HpIgG and $5.26(0.2,15)$ for HpSAg. While in patient group the results were significantly different compared to control group ( $\mathrm{p}<0.01$ ) where only $37.5 \%$ exhibited positive HpIgA results with $1.728(1.2,2.1)$, the percentage increased in HpIgG results where $87.5 \%$ showed positive results with $1.991(1.2,3.8)$ and reached its maximum 96.7\% in HpSAg results with 103.162 (20.3, 250) ng/ml as shown in figure 1 .

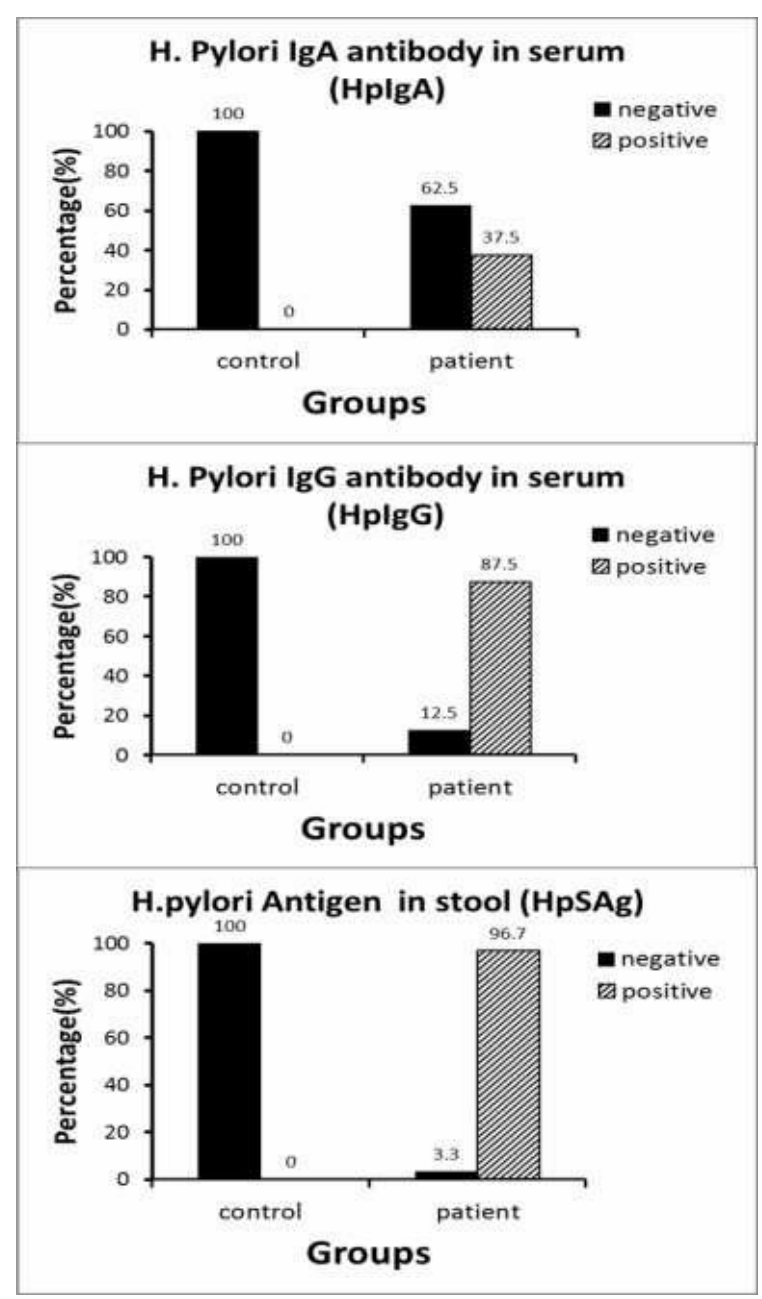

Fig. 1: Diagnostic tests to detect Helicobacter pylori infection
The overall sensitivity, specificity, and positive and negative predictive values are given in Table 3 . As expected all the tests gave $100 \%$ specificity and PPV. The most sensitive methods were observed in HpSAg, while the serological tests gave lower sensitivity results than HpSAg. In general the HpIgG was more sensitive than HpIgA test. The differences in performance of the tests were not related to sex as there was no significance difference in all tests between the different genders ( $\mathrm{p}>$ 0.1 ) as seen in figure 2 .

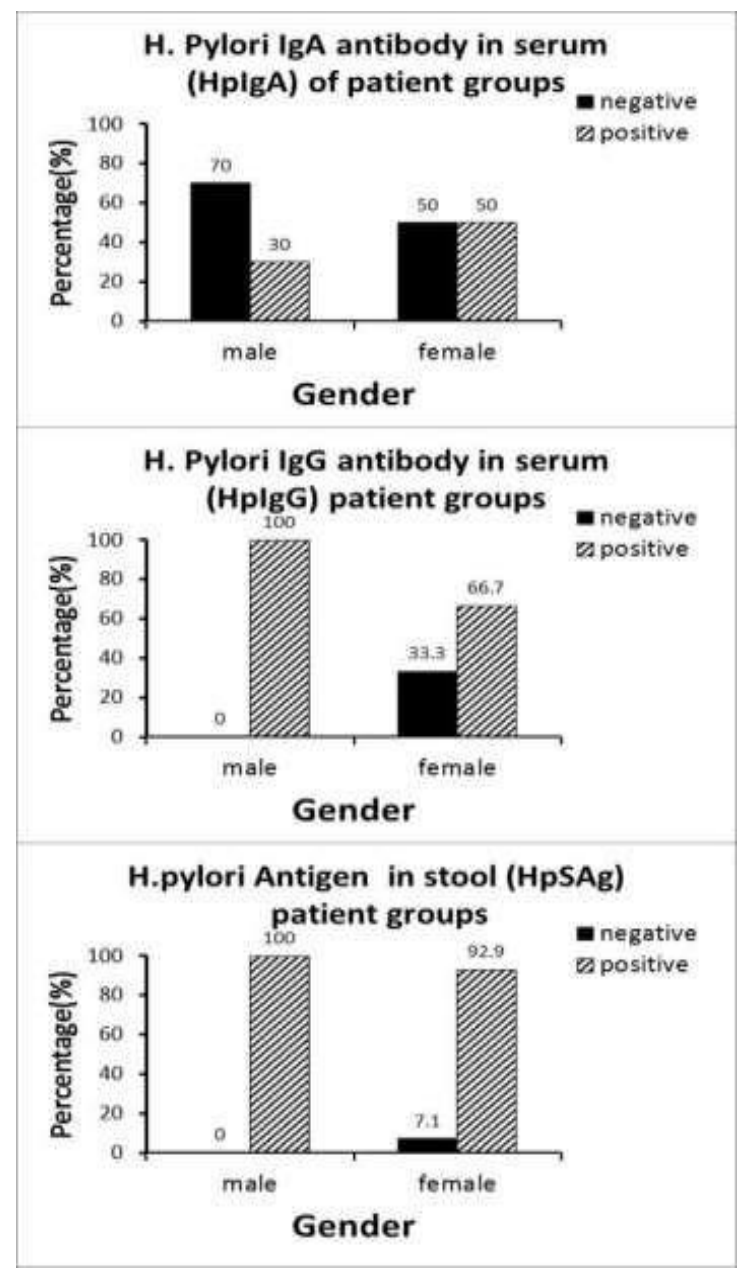

Fig. 2: The differences in results between male and female patients for Helicobacter pylori infections' detection tests. 
Table 3: Performance of Helicobacter pylori infections' detection tests

\begin{tabular}{|c|c|c|c|c|}
\hline Tests & Sensitivity (\%) & Specificity $(\%)$ & PPV (\%) & NPV (\%) \\
\hline HpIgA & $37.50 \%$ & $100.00 \%$ & $100.00 \%$ & $66.67 \%$ \\
& $(15.20 \%$ to $64.57 \%)$ & $(83.16 \%$ to $100.00 \%)$ & $(54.07 \%$ to $100.00 \%)$ & $(47.19 \%$ to $82.71 \%)$ \\
\hline HpIgG & $87.50 \%$ & $100.00 \%$ & $100.00 \%$ & $90.91 \%$ \\
& $(61.65 \%$ to $98.45 \%)$ & $(83.16 \%$ to $100.00 \%)$ & $(76.84 \%$ to $100.00 \%)$ & $(70.84 \%$ to $98.88 \%)$ \\
\hline HpSAg & $96.67 \%$ & $100.00 \%$ & $100.00 \%$ & $95.24 \%$ \\
& $(82.78 \%$ to $99.92 \%)$ & $(83.16 \%$ to $100.00 \%)$ & $(88.06 \%$ to $100.00 \%)$ & $(76.18 \%$ to $99.88 \%)$ \\
\hline
\end{tabular}

Values in parentheses are $95 \%$ confidence intervals

A significant difference between the results of both serological tests (HpIgG and HpIgA) was noted as the sensitivity of HpIgG was significantly higher than HpIgA ( $\mathrm{p}<0.001)$. The same result was observed between HpSAg and HpIgA as the results of HpSAg were significant more sensitive than HpIgA ( $p<0.001$ ), while the difference between HpIgG and HpSAg were equivalent ( $p>0.1)$.

\section{Discussion}

H. Pylori infection develop no symptoms in about $85 \%$ of the infected patient, however acute infection may appear as an acute gastritis with abdominal pain (stomach ache) or nausea which are quite broad and confusing symptoms especially for elder people. Knowing that the rate of infection increases with age and the infection tends to be common where sanitation is poor or living quarters are cramped as observed in the developing countries of the world. The infection remains localized to the gastric area, and probably persists unless specific treatment is given which may lead to peptic ulcer disease and gastric cancer to around $15 \%$ of the patients (Salih 2009, Bytzer , 2011). Early diagnosis increases the chances to eradicate it and allow the ulcer to heal rapidly. Many tests could be used to detect H. Pylori divided into invasive and noninvasive techniques based upon the need for endoscopy. Commonly the first noninvasively testes used especially in developed countries are the most simple and least expensive method by immunological tests like blood antibody test and stool antigen test formed by provincial health laboratories. Thus, in this study we evaluated the most common requested diagnostic tests to detect $\mathrm{H}$. Pylori infection in Egypt. We investigated the most efficient susceptibility immunological test for early detection of H.pylori infection by comparing the results of H. Pylori IgA, IgG in serum blood and H.pylori Ag in stool for 30 patients (males and females), age (30-60 years) against control group using conventional ELISA test.

In our study serological tests (HpIgA and HpIgG) gave average sensitivity results with mean above $50 \%$ (62.5\%), average NPV $78.80 \%$, however they exhibited $100 \%$ specificity and PPV with clear distinctive results as there was no indeterminate readings. Our results are in consistent with different studies reported the performance of serological tests especially HpIgG where the sensitivity and specificity have found uniformly high sensitivity but variable specificity ranging from 30-100\%12-15.The results of HpIgG were significant higher than that observed in HpIgA which are different from other studies observed HpIgA to be equal to HpIgG in performance with no additional benefit (Best, et al, 1994, Kindermann , et al, 2001,). However in other study the sensitivity of HpIgA was inversely proportional with age as poor sensitivity increased with adults ( $>18$ years old) than children ( She , et al, 2009). Interestingly only $25 \%$ of the patient group showed positive results in both HpIgG and HpIgA, while $12.5 \%$ showed only positive HpIgA and 62.5\% gave positive HpIgG only. Also there was no significant association between the results of HpIgG and HpIgA in all subjects. The same observation was reported in different studies showing presence of IgA antibody in absence of IgG and vice versa ( Jaskowski et al, 1997, Shirin 1999). These findings strongly suggested that HpIgA may not be the wise choice to be used for detection of H. Pylori infection in elderly people. Also the presence of positive HpIgA indicates a positive confirmation for early stage of infection or recurrence even in absence of positive HpIgG which was found previously in other studies (Veenendaal , 1991,Morris , et al, 1991, Aromaa , 1996) observed the predominantly IgG immune response to infection with $\mathrm{H}$. pylori which explained why the mean of values for HpIgG produced in patient group was 
significantly higher than HpIgA (0.948 and 1.798) respectively.

The Helicobacter pylori stool antigen test (HpSAg) is an enzyme immunoassay which detects H.pylori antigens present in human stool samples for diagnosis, monitoring effectiveness of antibiotic therapy during the 14 days of treatment and for confirmation of eradication. HpSAg is FDA approved and recommended by both the American Gastroenterological Association (AGA) and the American College of Gastroenterologists (ACG) (Talley 2005, Chey and Wong, 2007). HpSAg offers simpler sampling method; only one stool specimen is required and it does not require neither a technician nor nurse nor expensive equipment which make if suitable for children and Patients With Belonephobia. Comparing to serology tests, HpSAg is rapid, easy-to-use, non-invasive and had no confounding factors. Besides

HpSAg differentiate between active and latent infection; whereas, serology only detects exposure. Also large studies confirmed the high specificity and sensitivity of the test (Fanti, 1999,Vaira et al. 1999, Ohkura, 2000). In our study the results were consistent to the previously observation. HpSAg give the highest sensitivity and NPV (> 95\%) which is significant higher than HpIgA but equivalent to the HpIgG. Also there was no significant association between the results of $\mathrm{HpSAg}$ and both serological tests (HpIgG and HpIgA) in patient group only. As only $37.5 \%$ from patient group exhibited positive results in both HpSAg and HpIgA which represented all the positive results in HpIgA test. We could conclude that HpIgA is strong positive confirmatory test if appeared but could not be used alone as sole detection test. While $81.25 \%$ gave positive results in both HpSAg and HpIgG and 56.25\% of patient group showed positive HpSAg but negative HpIgA. Only one patient had negative results of both HpSAg and HpIgA but positive HpIgG which may refer to past infection or ongoing curing. Also $12.5 \%$ of patients group showed positive HpSAg, HpIgA and negative HpIgG results. Again as explained before these results may refer to early stage of infection. The overall findings confirmed the observation that serological tests are marker for infection rather than an indicator for active infection. Opposite results were observed when the results of all the subjects including (both control and patient groups) were compared. There was significant relation between the results of HpSAg and either HpIgA or HpIgG ( $\mathrm{p}<0.01)$ which was due to the common negative results in all control groups. These suggested that all the tests were good indicator for absence of $\mathrm{H}$. Pylori infection if combined together. In other words if patient gave negative results in two of the three tests one of them (HpSAg), this means that the chance to have H. Pylori infection is negligible even if he exhibited similar symptoms.

\section{Conclusion}

Given the widespread prevalence of H.pylori infection, its socioeconomic impact, and the rising antibiotic resistance rates worldwide. This investigation was performed to draw attention of the physicians to the value and importance of immunological testing as noninvasive, relatively cheap easy to be performed and convenient to patients for the recognition of H.pylori infection. Collectively our results suggested their utility as a reliable predictor of infection in high-prevalence developing country especially $\mathrm{HpSAg}$ test which exhibited best results to be the first choice to detect early infection followed by HpIgG as confirmatory test in adult patients, while HpIgA could not be used alone for diagnosis of H.pylori infection.

\section{References}

Atherton, J. C. \& Blaser, M. J.(2009). Coadaptation of Helicobacter pylori and humans: ancient history, modern implications. The Journal of clinical investigation119: 2475 .

Aromaa, A., kosunen, TU., Knekt, P., Maatela, J., Teppol, Heinonen, O. P., Harkonen, M., Hakama, M. K.(1996). Circulating anti-Helicobacter pylori immunoglobulin A antibodies and low serum pepsinogen I level are associated with increased risk of gastric cancer. American J epidemiology144:142-149 .

Blaser, M. J. (2006). Who are we? Indigenous

microbes and the ecology of human diseases. EMBO reports7, 956 .

Bardhan, P. K. (1997). Epidemiological features of Helicobacter pylori infection in developing countries. Clinical infectious diseases25: 973-978.

Bytzer, P., Dahlerrup, J. E.,Jarbol, D. E., Rosenstock., S.,Wildt, S., (2011).Danish society for Gastroenterology.Diagnosis and treatment of Helicobacter pylori infection. Dan Med Bull, C427 .

Best, L. M., Van Zanten, S. V., Sherman, P. M. \& Bezanson, G. S.,(1994). Serological detection of Helicobacter pylori antibodies in children and their parents. Journal of clinical microbiology32: 1193-1196 . 
Chey, W. \& Wong, B.,(2007). Practice Parameters Committee of the American College of Gastroenterology. American College of Gastroenterology guideline on the management of Helicobacter pylori infection. Am J Gastroenterol. 102: 1808-1825 .

El Dine, S. S., Mubarak, M., Salama, R., El Raziky, M., El Sherbiny, E., Zakaria, S., \& Serag, M., (2008). Low Seroprevalence of anti-CagA antibodies inspite of high seroprevalence of anti-H. Pylori antibodies in rural Egyptian community. Research Journal of Medicine and Medical Sciences 3:118-23 .

Frenck, R. W. \& Clemens, J., (2003). Helicobacter in the developing world. Microbes and infection. 5: 705713 .

Fanti, L., Mezzi, G., Cavallero, A., Gesu, G., Bonato, C. \& Masci, E., (1999). A new simple immunoassay for detecting Helicobacter pylori infection: antigen in stool specimens. Digestion. 60: 456-460

Goossens, H., Glupczynski, Y., Burette, A., Borre, C. Van den \& Butzler, J.,(1992). Evaluation of a commercially available second-generation immunoglobulin G enzyme immunoassay for detection of Helicobacter pylori infection. Journal of clinical microbiology.30:176180 .

Jaskowski, T., Martins, T., Hill, H. \& Litwin, C., (1997). Immunoglobulin A antibodies to Helicobacter pylori. Journal of clinical microbiology.35: 2999-3000.

Kusters, J. G., Vliet, A. H. \& Kuipers, E. J., (2006). Pathogenesis of Helicobacter pylori infection. Clinical microbiology reviews.19:449-490 .

Kindermann, A., Konstantopoulos, N., Lehn, N., Demmelmair, H. \& Koletzko, S., (2001) .Evaluation of Two Commercial Enzyme Immunoassays, Testing Immunoglobulin G (IgG) and IgA Responses, for Diagnosis ofHelicobacter pylori Infection in Children. Journal of clinical microbiology. 39:3591-3596 .

- Marshall, B.J. \& Warren, J. R., (1984). Unidentified curved bacilli in the stomach of patients with gastritis and peptic ulceration. The Lancet 323: 1311-1315

Marshall, B., Stenström, B. \& Mendis,A., (2008)

.Helicobacter pylori: the latest in diagnosis and treatment. Australian family physician, 608 .

Morris, A. J., Ali, M. R., Nicholson, G. I., PerezPerez, G. I. \& Blaser, M. J., (1991) Long-term follow-up of voluntary ingestion of Helicobacter pylori. Annals of internal medicine.114: 662-663 .

Ohkura, R., Miwa, H., Murai, T., Nagahari, A., Ohta,k., Sato,k., Yamada,T. \& Sato,N., (2000). Usefulness of a novel enzyme immunoassay for the detection of Helicobacter pylori in feces.Scandinavian journal of gastroenterology. 35:49-53 .

Rathbone, B. J., Wyatt, J. I.,\& Heatley, R. V., (1985). Immune response to Campylobacter pyloridis. The Lancet, 1217 .

Salih, B. A.,(2009). Helicobacter pylori infection in developing countries: the burden for how long? Saudi J Gastroenterology,15:201-207 